\title{
Analysis of Physicochemical Natures of Modern Artifacts in MRI
}

\author{
https://doi.org/10.3991/ijoe.v18i03.25859
}

Yaroslav Volodymyrovych Tsekhmister ${ }^{1(\bowtie)}$, Viktor Ivanovich Stepanenko², Tetiana Konovalova ${ }^{2}$, Bogdan Yaroslavovych Tsekhmister ${ }^{1}$

${ }^{1}$ Ukrainian Medical Lyceum, O.O. Bogomolets National Medical University, Kyiv, Ukraine ${ }^{2}$ O.O. Bogomolets National Medical University, Kyiv, Ukraine

$$
\text { ya_tsekhmister@ukr.net }
$$

\begin{abstract}
Magnetic resonance imaging (MRI) is very useful in the medical sector as it uses radio waves and robust magnetic fields to produce images of the inside of the human body. MRIs are so helpful in pathologies. However, artifacts in MRI occur due to the complex nature of MRI. Artifacts in MRI may reduce the examination quality and may also be confused with pathology. Failing to identify artifacts in MRI can bring troubles. Hence, it's very important to reduce or avoid artifacts in MRI. The major reasons behind artifacts in MRI are improper action of the MRI scanner, magnetic susceptibility, motion, chemical shifts, variation in signal intensity, etc. Due to MRI artifacts, patients can suffer from serious problems. Therefore, we have analyzed the physicochemical natures of artifacts in MRI. The research has made attempts to identify various types of artifacts in MRI and find out the causes of these artifacts. There have been samples of various types of artifacts based on which the analyses have been done. Based on these identifications of artifacts, there have also been discussions and analyses on how to avoid artifacts in MRI.
\end{abstract}

Keywords - magnetic resonance imaging, computerized tomography, artifact, magnetic susceptibility

\section{Introduction}

Open the document you would like to format and import the styles. How this works depends very much on the version of MS WORD that you use. The styles' names to be used for online-journals.org are preceded by a "0_" which makes them appear first in the styles list and therefore easier to be found.

Now just place the cursor in the paragraph you would like to format and click on the corresponding style in the styles window (or ribbon). 


\section{$2 \quad$ Literature review}

There has been a wide range of research on artifacts in MRI. Artifacts in MRIbased CT can badly degrade proton therapy's dose calculation accuracy. Inspired by the efficiency of MRI in radiation therapy, there was a development of an artifact correcting method in MRI-based CT. The method makes the proton range calculation more accurate [3]. The correction procedure ensured impressive artifact reduction, both qualitatively and quantitatively. After correction, mean error was reduced in simulated brain and neck and head CT images. There's more research on MRI, such as the TQM principles-based interventions for improving report generation and delivery process [4].

It's possible to evaluate needle artifacts' characteristics and needle insertion's accuracy by using a 1.2 Tesla open MRI system in a phantom [5]. To evaluate artifacts, various needle phenomena are examined with different needle angles varying from $0^{\circ}$ to $90^{\circ}$. These tests were able to clarify needle artifacts' characteristics in 1.2 Tesla open MRIs. The developed system can also ensure an acceptable accuracy of needle insertion guided by MRI fluoroscopy in clinical use. MRI-based research has also simplified diagnosing post-operative complexities after implant surgery. Due to susceptibility artifacts in data scanning, the uses of gradient-echo sequences and increasing field strengths have highlighted difficulties [6]. Artifacts can arise around metal implants, including implants made of titanium alloys. These artifacts create problems to detect complications such as infection, bleeding, etc., and hinder surrounding structure's imaging such as inner ear, brain, etc. The developed upstream design uses lightweight structures to manage artifacts. While implemented on representative cranioplasty, artifact reduction was $10 \%$ more as compared to traditional solid material.

For MRI of lungs, PROPELLER (Periodically rotated overlapping parallel lines with enhanced reconstruction, Multivane, MV) technique has been very effective [7]. The research compared T2 Fast Spin Echo (FSE) and T2-MV at 1.5T. A four-point scale rated image quality and artifacts. Also, low-dose CT was correlated to lesion detection. The MV sequence obtained from the research showed fewer motion artifacts and pulsation and better image quality than FSE. MV sequence also ensured higher pulmonary detection rates. MV also ensured higher specificity for larger lesions. These experiment results proved that MV is extremely efficient for strong artifact reduction and superior image quality.

MRI parameters have been able to influence the characterization and optimal detection of small vessel disease (SVD) while investigating patients with the possibility of cerebral amyloid angiopathy (CAA) in the context of cognitive dysfunction or intracranial hemorrhage (subarachnoid or parenchymal). CAA is a frequent age-related SVD. Cardinal MRI signatures of CAA are hemorrhagic in nature [8]. CAA also influences the presence of intracerebral hemorrhages, strictly lobar cerebral microbleeds, and cortical superficial siderosis. In clinical practice, MRI techniques have successfully detected major hemorrhagic CAA imaging signatures. The research utilized the imaging parameters while encountering CAA patients.

MRI features provide good results in post-traumatic osteomyelitis (PTOM) with metal implants using a machine of 3.0T with protocols of conventional metal artifact reduction (MAR). MR diagnosis showed good results in specificity, sensitivity, positive and negative values. While diagnosing PTOM of the extremities by 3.0T MRI with 
conventional parameters of metal artifact reduction, the accuracy has been $71 \%$, which is very high as compared to the accuracies determined by existing works [9].

While differentiating among aseptic loosening, periprosthetic joint infection (PJI), and cases without any pathology after total hip arthroplasty (THA), MRI with metal artifact reduction (MAR) provides image characteristics efficiently [10]. There were specific findings at the ace tabular cup for either PJI or aseptic loosening. At the stem, the findings were sensitive. Soft tissue edema, ace tabular and femoral components, enlarged lymph nodes, and abnormalities were accurate; therefore, differentiating aseptic loosening and PJI was effective. For therapy planning, these findings have been very helpful.

3.0T MRI has characterized artifacts' levels and titanium applicators' distortions effectively. In that research, T1-weighted MRI with a slice of $1 \mathrm{~mm}$ thickness was a favorable MR sequence. Artifact width at the tandem's tip was much lesser than those of existing tests.

In MRI of the human brain, there may be artifacts in head motion estimates due to fluctuations of the main magnetic field caused by body motion. MRI (rs-fcMRI) with multiband resting-state functional connectivity examines head motion effectively. The method using a band-stop filter removes respiratory effects from head motion estimates [11]. Thus artifacts in head motion estimates can be controlled. The band-stop filter improves the quality of post-processing fMRI data.

MRI techniques have been effective in the classification of tumor grades from magnetic resonance. This research has explained methods such as classifying brain tumors, screening, processing, tissue extraction, tumor's statistical features using Flair brain MRI and two types of T1W, etc. This approach has successfully increased brain tumor diagnosis's reliability [12]. MRI has also been effective in detecting the human brain's uncommon portions. The research obtained $98 \%$ accuracy in detecting tumor or human brain's abnormality [13]. For classifying brain MRIs, a biologically inspired framework based on Extreme Learning Machine (ELM) is very effective [14].

There have been researches on reducing the processing time of the reconstruction of MRI signals [15]. This research worked with many samples needed for reconstruction of MRI to reduce processing time and increase scanning speed.

\section{Types of artifacts in MRI}

There are various types of artifacts in MRI, such as physiological artifacts, artifacts due to physical phenomena (susceptibility, chemical shift, etc.), artifacts due to equipment failure, artifacts due to operators' incorrect actions, etc.

\subsection{Physiological artifacts}

Intentional or unintentional patient motion can create physiological MRI artifacts. Motion artifacts typically arise as ghosting or blurring, especially in the direction of phase encoding. Patients hold still in the exams as motion can make images complex. But there will still be some breathing, blood flow, and heartbeats during an exam. So, artifacts may arise and make the sequences and respiratory-ordered phase encoding difficult [16]. 


\subsection{Artifacts due to physical phenomena}

Artifacts due to chemical shifts occur during the MRI process's frequency encoding. Within the magnetic field, water and other fat molecules oscillate differently; therefore, these molecules appear differently in the encoding stage. So, at the anatomy's edge, a bright or black band arises. MRI experts often misunderstand these as pathologies. Due to magnetic susceptibility, artifacts may arise during the implant of a patient or during the response of a medical device to the magnetic field in the MR room. These materials cause dark and bright areas around the magnetic object in the image. These magnetic objects aren't anatomically correct.

\subsection{Artifacts due to equipment failure or operators}

Zipper artifacts cause noise that makes the reading of images difficult. When there's an interference between the MRI unit and something in the environment, such an incident occurs. Some examples include devices in the MR room, the door improperly shut, blinking light bulbs, etc. Operators can also be responsible for artifacts if they make faults during MRI.

\section{$4 \quad$ Examined artifacts and their physicochemical natures}

The research includes examinations of various MRI samples. There have been identifications of various artifacts along with their natures. This section will explain various artifacts and their natures.

\subsection{Artifacts caused by magnetic susceptibility}

Magnetic susceptibility artifacts are like spatial distortions or bright spots [17]. These artifacts arise due to changes in field strength or microscopic gradient near the substances' surfaces with different magnetic susceptibility. Medical devices inside or near the display area can cause these artifacts. Strong artifacts often arise near ferromagnetic objects in diamagnetic materials (as an example, the human body). In the spins' phase and the surrounding tissues' frequencies, there's a shift due to these gradients. Such a shift leads to dark and bright areas around the tissues with spatial distortions. A fabric's magnetic susceptibility indicates the capability of the fabric to be magnetized. Most tissues' susceptibility values are in a narrow range. There are local changes in magnetic susceptibility due to the presence of "air/tissue" boundaries or ferromagnetic substances such as enhanced local concentration of hemoglobin during the use of ferromagnetic contrast agents or during hemorrhage. As a result, in the magnetic field, there's an impaired local homogeneity, as shown in Figure 1. Figure 1 shows susceptibility artifacts because of particles of iron oxide used as oral contrast agents. There was a distortion of the local magnetic field due to the substance's very high concentration. The distortion led to the appearance of artifacts.

There are different ways to detect susceptibility artifacts based on local conditions. It's possible to decrease or increase the signal intensity. The boundaries responsible 
for the artifacts are somewhere in the lungs, around the nasopharynx, and with extra sinuses. The presence of air in the lungs causes the lack of a signal. Susceptibility artifacts are also responsible for the lack of a signal.

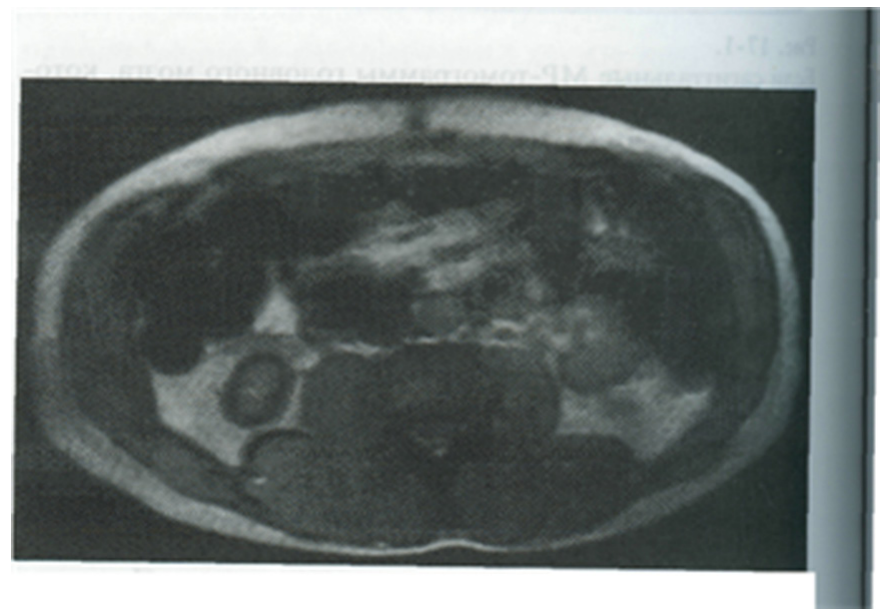

Fig. 1. Artifacts due to magnetic susceptibility

Instead of using gradient sequences, it's easier to eliminate susceptibility artifacts by using a spin-sequence echo. If there's a local change in the magnetic field, it's possible to determine the change using a special pulse sequence or phase images. This procedure builds the local field's map based on the two signals' interaction. Susceptibility artifacts may occur due to contrast agents during contrast-enhanced MR angiography. By pronouncing these artifacts, it's possible to reduce these by scanning the whole normalized space, i.e., not following the partial scanning techniques in the normalized space.

\subsection{Artifacts caused by respiratory movements and heart contractions}

In MRI, the most common artifacts are motion artifacts. Studying the abdominal cavity becomes difficult due to motion artifacts. Because of these artifacts, obtaining thoracic organs cells' images has to be synchronized with heartbeats. Due to the respiratory movement, anatomical structures' boundaries become blurred, and additional ghostly images occur. The blurring is due to the image averaging of a moving organ or structure; it's similar to a moving object's photograph with short exposure. It can result in the enticing of small pathological foci. Ghost images are the main image's partial copies appearing in different places. Most often, a flow of fluid causes ghost images. Figure 2 shows artifacts or ghost images due to heart contractions and respiratory movements. The motion's direction is along the phase-coding gradient in both cases. In the chest, respiratory movements cause many separate ghostly images, and the heartbeat is responsible for a column of noise that passes through the heart. The movement can be between different lines' receipts in the normalized space. Artifacts due to this movement are avoidable by synchronizing data collection with physiological methods. Another movement is between the data acquisition and the excitable RF pulse. 


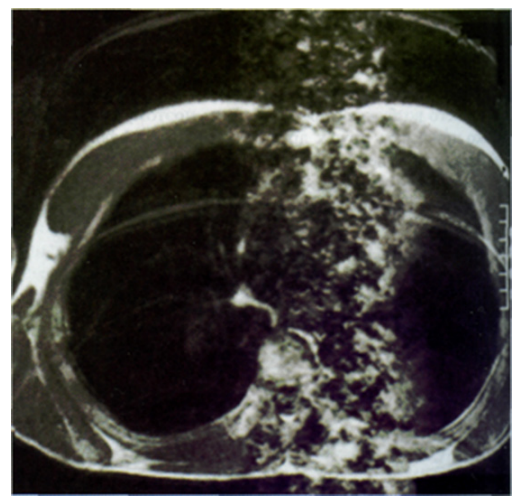

Fig. 2. Artifacts due to respiratory movements and heart contractions

\subsection{Artifacts caused by flow}

Artifacts' origins due to flow are similar to artifacts' origins due to movement. The movements of Cerebrospinal fluid (CSF) and blood are pulsating. Therefore, in various rows of normalized space, there will be different flow rates. In the current substance, there are phase shifts due to reading and selection layer gradients. From streams, artifacts can be like general blur or individual artifacts in the phase-coding direction. Blood may depict outside the vessels due to the signal's incorrect registration. Flow artifacts can occur in iliac vessels, femoral vessels, popliteal vessels, etc. [18]. Figure 3 shows the neck's gradient tomogram. Artifacts caused by flow are like noise columns moving through arterial vessels' locations. Venous blood flow is also responsible for artifacts, but it's not much pronounced as the veins' pulsation is weaker than the pulsation of the arteries. From fluxes, artifacts are especially pronounceable on gradient tomograms, according to Figure 6. It's because the current spins weren't in exposure to previous RF pulses while doing two-dimensional scanning. Therefore, the magnetization is fully restored. This results in a highly intense signal from the current blood. It means there are pronounced artifacts while there aren't any compensating gradients or synchronization.

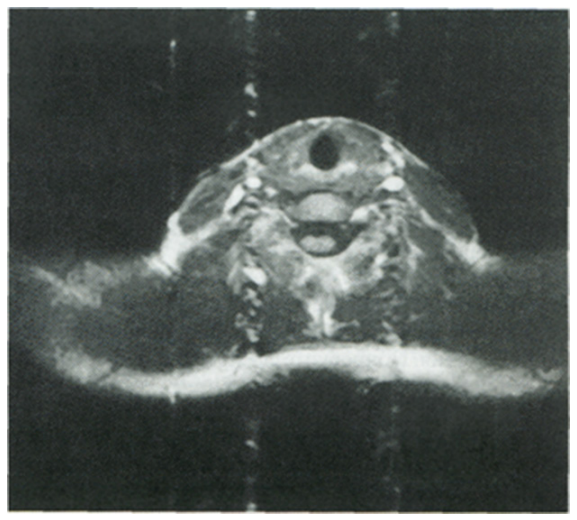

Fig. 3. Artifacts due to flow 


\subsection{Artifacts caused by chemical shift}

Variation in protons' resonant frequencies in various chemical environments is mainly responsible for chemical shift artifacts. The resonant frequencies of water protons and adipose tissue protons vary by three parts per million. Slice coding and frequency coding processes use resonant frequency information. Due to the variation of signals of water protons and adipose tissue, the frequencies differ, and so there's a shift in the images relative to each other. Figure 4 shows this case. The artifact relies on the resonant frequency, and its impact is the most in a strong magnetic field when there's a shift of signals by some pixels in the reading direction. It's possible to control the artifact by strong gradient fields. In Figure 4, the MR tomogram is obtained in a robust magnetic field of $1.5 \mathrm{~T}$. These tomograms show artifacts due to chemical shifts in the direction of reading (corresponds to vertical in the image in Figure 4). The artifact is like a black outline between muscle and adipose tissue. The problem is avoidable if we can suppress the signal from any of the components before the collection of every row of data. For this, there are methods such as pressure which needs the constant magnetic field's high homogeneity. Another way is one of the schemes of adding and subtracting.

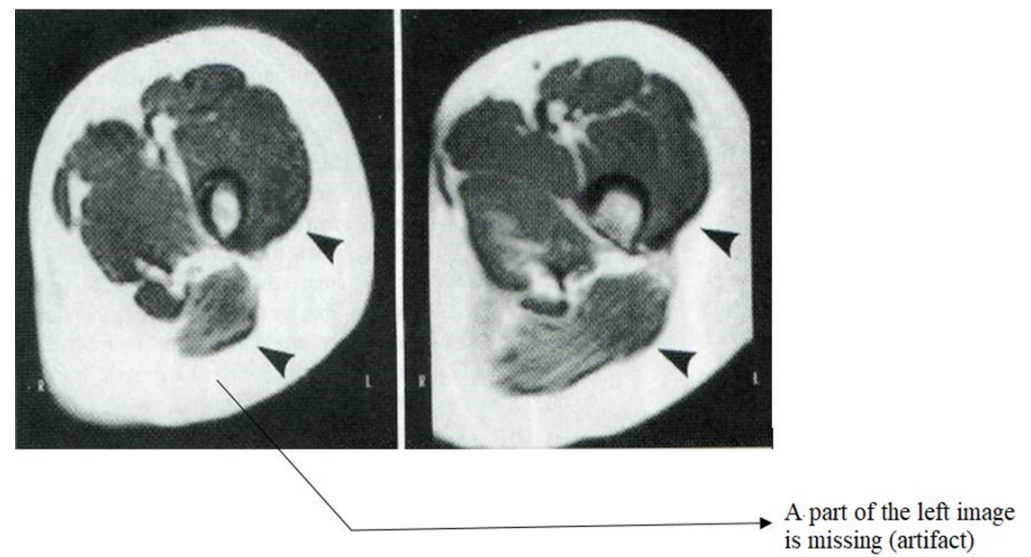

Fig. 4. Artifacts due to chemical shifts

\subsection{Artifacts of the black border}

Sometimes there are visible clear black contours near anatomical structures. These artifacts are also due to chemical shifts. This artifact is like a black line at the two medias' (fat muscle/fat/water) boundary. Signals from adipose tissue and water may be in antiphase or phase. This type of artifact's appearance is because of the wrong choice of TE during the mutual compensation of the spins of fat and water in antiphase. These artifacts appear while using inversion-recovery sequences or gradient sequences. Figure 5 shows black border artifacts during the abdominal organs' study using gradient sequences. Here, the gradient pulse sequence is with a TE of $16 \mathrm{~ms}$. To remove this artifact, it's necessary to use recommended TE values when the signals from adipose tissue and water are in the same phase. Another way is using a spin-moon sequence with $180^{\circ}$ pulses refocusing phase shifts while conducting research. 


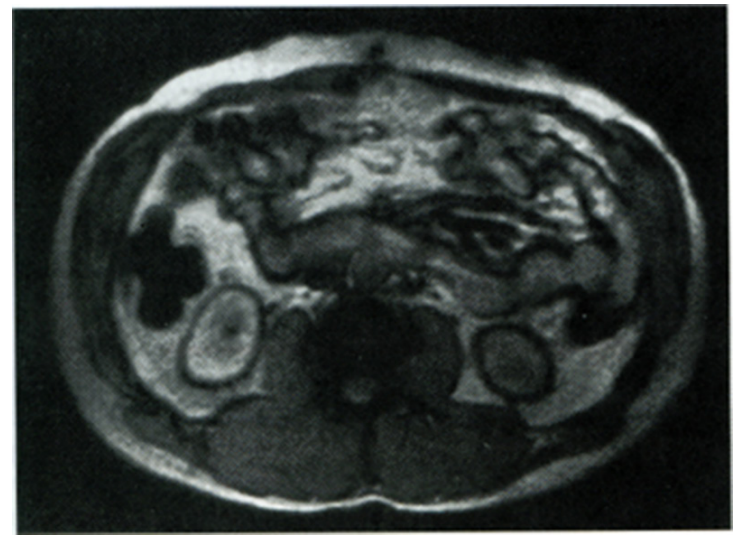

Fig. 5. Artifacts of the black border

\subsection{Truncation artifacts}

This type of artifact manifests itself in the parallel bands' form near or around the border between tissues with various signal intensities (e.g., muscle or cerebrospinal fluid/ adipose tissue/ spinal cord). The lines resemble genuine formations, so if these stay unnoticed as artifacts, there can be difficulties in the interpretation of images. These artifacts are strong while using a small matrix. So, it's possible to eliminate these by using a bigger matrix [19]. A larger number of averages doesn't affect the truncation artifacts' intensity but reduces the gap between the lines. Therefore, the artifacts become invisible and more blurred.

Truncation artifacts often are in the phase-coding direction. In this direction, a larger size of the matrix results in an undesirable increase in study time. The selection of suitable orientation of data collection and increasing the matrix's size in the direction of frequency-coding usually help to reduce artifacts to a low level. Figure 6 shows such an artifact and its reduction to an acceptable level. It's also called Gibbs' bell. However, a low-pass filter can reduce truncation artifacts, but with every type of normalized space filtering, this process will affect the entire image.
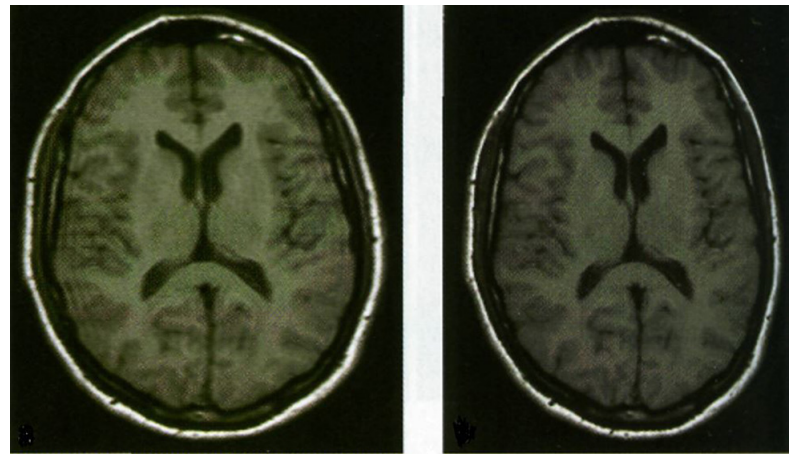

Fig. 6. Truncation artifacts 


\subsection{Artifact overlay}

Sometimes, the data outside of the selected field starts to be used for constructing the image; thus, overlay artifacts form. This can happen in both frequency-coding and phase-coding directions. In the frequency-coding direction, the reason for this artifact is the presence of high-frequency signals. These signals are erroneously located in the normalized space. In order to properly reproduce frequency information, it has to be collected twice.

Redundant data may stay on both its opposite and own side based on the signal registration scheme. Using a filter, it's possible to eliminate high-frequency signals. But all filters don't match the expected frequency range or the image frequency band. Therefore, some artifacts still exist, or there's a signal loss at the image's edge. It's possible to overcome this problem by the double collection of data. For this, either tomography speed to the frequency of critical data acquisition needs to be double, or the image acquisition time needs to be double. The latter option has more advantages as it ensures a double signal to noise ratio per square root. Then we need a filter for removing all frequencies absent in the new image's frequency band. However, the selected image field's frequencies will stay free of any impact due to this action. After the Fourier transformation, the external data obtained from the doubling of data collection is discarded, and the image field becomes free of artifacts. Figure 7 is an example of this process. It's a tomogram of kiwi fruit. The left image is the normal image, and the right image is the image with double the amount of data collection. In both cases, the frequency-coding gradients are in the vertical direction. In the left image, the signal's overlay from outside the selected field of view results in an artifact's appearance.

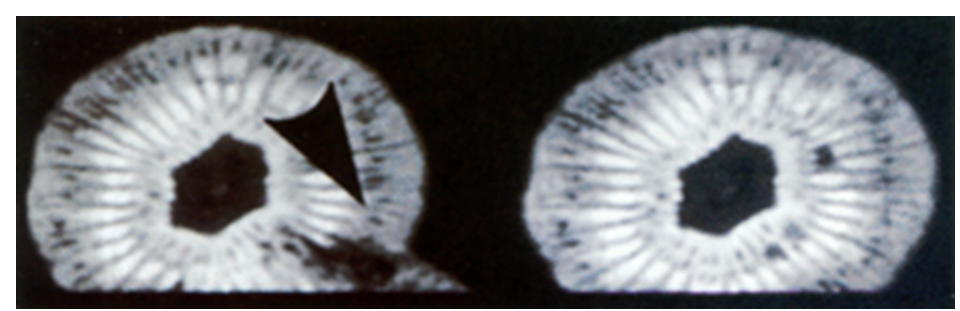

Fig. 7. Overlay artifacts

\subsection{Quadrature artifact}

Figure 8 shows an example of a quadrature artifact. A receiver with two channels records the MR signal. In the second channel, there's a shift of the signal by exactly $90^{\circ}$ in phase relative to the first channel. If the phase shift is improper, there will be an erroneous image inverted around the two ( $\mathrm{x}$ and $\mathrm{y}$ ) axes relative to the main image. It's important to adjust the gain and phase of the receiver to eliminate the artifact. Also, in the converted extra-resonant signal, reducing the quadrature peak is important to ensure eliminating the artifact. 


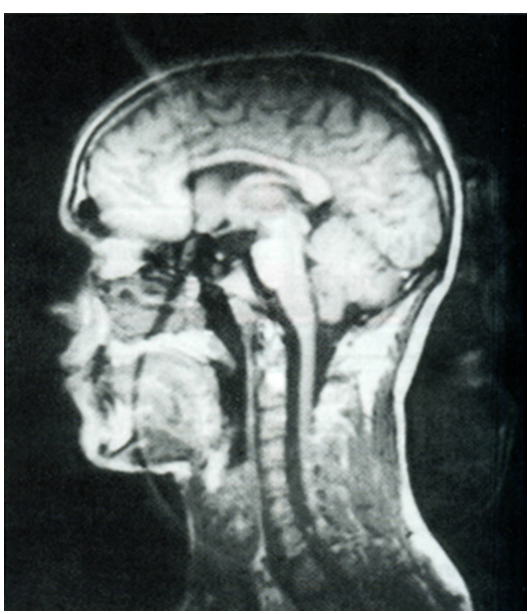

Fig. 8. Artifacts quadrature artifacts

\section{Discussion}

As observed from the MRI samples, artifacts can be of various types. MRI experts need to be careful while operating. For being careful with magnetic field based artifacts, it's important to check magnetic susceptibility, ferromagnetic substances, signal intensity, etc. There are effective ways to eliminate artifacts due to magnetic fields. Artifacts due to respiratory movements or flows can make images blurred. Also, ghost images can appear. These artifacts can be controlled by synchronizing data collection, controlling tomography time, etc. For avoiding chemical shift artifacts, it's important to check resonant frequencies. The use of robust gradient fields can reduce chemical shift artifacts. Also, suppression of signals effectively reduces chemical shift artifacts. For black border artifacts, it's necessary to check if there are unexpected black lines in boundaries among water, fat, fat muscles, etc. In the case of truncation artifacts, applying a low pass filter can be effective in eliminating the artifacts. While using a small matrix, truncation artifacts often occur. Another type of artifact is overlay artifact which can be reduced by doubling the collection of data. Another artifact from our finding was the quadrature artifact which can be controlled by adjusting the receiver's gain and phase and minimizing the quadrature peak.

\section{Conclusion}

MRI is very important in the diagnosis of patients. Artifacts increase complexities in MRI studies. Therefore it's essential to know about various types of artifacts and the processes of identifying and reducing these artifacts. Every type of artifact has specific signs; therefore, knowing the details of various types of artifacts can help a lot in reducing artifacts. We hope this research will help MRI experts to know about artifacts and artifact-reducing techniques. 


\section{$7 \quad$ References}

[1] P. Lam, "MRI scans: Definition, uses, and procedure," Medicalnewstoday.com, 24-Jul2018. [Online]. Available: https://www.medicalnewstoday.com/articles/146309. [Accessed: 29-Jul-2021].

[2] K. Krupa and M. Bekiesińska-Figatowska, "Artifacts in magnetic resonance imaging," Pol. J. Radiol., vol. 80, pp. 93-106, 2015. https://doi.org/10.12659/PJR.892628

[3] P. C. Park et al., "MRI-based computed tomography metal artifact correction method for improving proton range calculation accuracy," Int. J. Radiat. Oncol. Biol. Phys., vol. 91, no. 4, pp. 849-856, 2015. https://doi.org/10.1016/j.ijrobp.2014.12.027

[4] P. Adikari, S. De Alwis, and L. Karunanayake, "Improving report generation and delivery system of microbiological investigations at MRI-Sri Lanka with concern to turn-aroundtime, an intervention study," Int. J. Onl. Eng., vol. 16, no. 09, p. 26, 2020. https://doi. org/10.3991/ijoe.v16i09.13799

[5] S. Okamoto et al., "Needle artifact characteristics and insertion accuracy using a 1.2T open MRI scanner: A phantom study," Diagn. Interv. Imaging, vol. 102, no. 6, pp. 363-370, 2021. https://doi.org/10.1016/j.diii.2020.12.007

[6] L. N. Carter et al., "Reducing MRI susceptibility artefacts in implants using additively manufactured porous Ti-6Al-4V structures," Acta Biomater, vol. 107, pp. 338-348, 2020. https://doi.org/10.1016/j.actbio.2020.02.038

[7] M. Meier-Schroers, G. Kukuk, R. Homsi, D. Skowasch, H. H. Schild, and D. Thomas, "MRI of the lung using the PROPELLER technique: Artifact reduction, better image quality and improved nodule detection," Eur. J. Radiol., vol. 85, no. 4, pp. 707-713, 2016. https://doi. org/10.1016/j.ejrad.2015.12.016

[8] G. Boulouis et al., "MRI for in vivo diagnosis of cerebral amyloid angiopathy: Tailoring artifacts to image hemorrhagic biomarkers," Rev. Neurol. (Paris), vol. 173, no. 9, pp. 554-561, 2017. https://doi.org/10.1016/j.neurol.2017.09.004

[9] B. N. Park, S.-J. Hong, M. A. Yoon, and J.-K. Oh, "MRI diagnosis for post-traumatic osteomyelitis of extremities using conventional metal-artifact reducing protocols: Revisited," Acad. Radiol., vol. 26, no. 11, pp. e317-e323, 2019. https://doi.org/10.1016/j.acra. 2019.01.001

[10] B. J. Schwaiger et al., "Diagnostic accuracy of MRI with metal artifact reduction for the detection of periprosthetic joint infection and aseptic loosening of total hip arthroplasty," European Journal of Radiology, vol. 131, no. 109253, p. 109253, 2020. https://doi. org/10.1016/j.ejrad.2020.109253

[11] D. A. Fair et al., "Correction of respiratory artifacts in MRI head motion estimates," Neuroimage, vol. 208, no. 116400, p. 116400, 2020. https://doi.org/10.1016/j.neuroimage. 2019.116400

[12] H. Samadi Ghoushchi and Y. Pourasad, "Clustering of brain tumors in brain MRI images based on extraction of textural and statistical features," International Journal of Online and Biomedical Engineering (iJOE), vol. 16, no. 12, p. 116, 2020. https://doi.org/10.3991/ijoe. v16i12.16929

[13] S. Lalitha, M. Katapally, K. Pabba, and V. Mudunuri, "Efficient tumor detection in MRI brain images," Int. J. Onl. Eng., vol. 16, no. 13, p. 122, 2020. https://doi.org/10.3991/ijoe. v16i13.18613

[14] S. P. Pati, D. Mishra, and S. Borah, "A biologically inspired ELM-based framework for classification of brain MRIs," International Journal of Online and Biomedical Engineering (iJOE), vol. 16, no. 10, p. 116, 2020. https://doi.org/10.3991/ijoe.v16i10.15653

[15] V. Upadhyaya and M. Salim, "To analyse the effect of relaxation type on magnetic resonance image compression using compressive sensing," International Journal of Online and Biomedical Engineering (iJOE), vol. 17, no. 04, p. 21, 2021. https://doi.org/10.3991/ijoe. $\underline{\text { v17i04.20759 }}$ 
[16] V. Harmonay, "Most common artifacts in MRI," Atlantisworldwide.com. [Online]. Available: https://info.atlantisworldwide.com/blog/artifacts-in-mri. [Accessed: 29-Jul-2021].

[17] U. Bashir, "Magnetic susceptibility artifact," Radiopaedia.org. [Online]. Available: https:// radiopaedia.org/articles/magnetic-susceptibility-artifact. [Accessed: 29-Jul-2021].

[18] Softways, "MRI Artifacts-flow artifact," Mr-tip.com. [Online]. Available: https://www. mr-tip.com/serv1.php?type=art\&sub=flow\%20artifact. [Accessed: 29-Jul-2021].

[19] "MR Image quality and artifacts," Imaios.com. [Online]. Available: https://www.imaios. com/en/e-Courses/e-MRI/Image-quality-and-artifacts. [Accessed: 29-Jul-2021].

\section{Authors}

Yaroslav V. Tsekhmister, Doctor of Science, Professor, Ed. D, PhD Degree Applicant, Ukrainian Medical Lyceum at O.O. Bogomolets National Medical University, Kyiv, Ukraine. Ukrainian scientist in the sphere of medical and biological physics, professional education, theory and teaching methodology, pre-occupational training for medical specialties, Candidate of Physics and Mathematics (1995), Doctor of Pedagogy (2002), Laureate of Bonus for Young Scientists in the sphere of physics by National Academy of Sciences of Ukraine (2001), professor (2003), Honorary Educator of Ukraine (2007), Master of Pharmacy (2013), Master of Public Law (2017), Professor of Education and Health Management (Honoris Causa), Open University of Liberal Sciences, USA (2017), Eastern European University Network, Lublin (2018), full member of the National Academy of Pedagogical Sciences of Ukraine (Department of General Pedagogy and Philosophy of Education, 2019). Pupil of Doctor of Physics and Mathematics, Professor, Honorary Scientist and Technician of Ukraine, corresponding member in National Academy of Pedagogy of Ukraine, head at Department of Medical and Biological Physics at O.O. Bogomolets National Medical University Oleksandr Vasyliovych Chalyi. Since 1, September, 2014 due to contest he was elected to the post of the first pro-rector in scientific pedagogical activity. According to the order from MHC of Ukraine, dd. 23.04.2018 No 18-O he was appointed to the post of acting rector (until 14, September, 2018). ORCID ID: 0000-0002-7959-3691 (email: ya tsekhmister@ukr.net). Scopus Author: https://www.scopus.com/authid/ detail.uri?authorId $=35747165900$

Viktor I. Stepanenko, $\mathrm{PhD}$ in Medicine Professor Head of the Department Of Dermatology and Venereology of Bogomolets National Medical University Kyiv, Ukraine, T. Shevchenka blvd., 13, 01601, Kyiv, Ukraine ORCID ID: 0000-0003-47101151 (email: stepanenkorl@ukr.net).

Tetiana S. Konovalova, MD, PhD, Professor (Associate) O.O. Bogomolets National Medical University, Kyiv, Ukraine, ORCID ID: 0000-0002-0319-9532 (email: tsc777@, ukr.net).

Bogdan Y. Tsekhmister, Chairman of the Scientific Society Scientific Society A.P. Romodanov of Ukrainian Medical Lyceum at O.O. Bogomolets National Medical University, Kyiv, Ukraine, ORCID ID: 0000-0002-7959-3691 (email: ya tsekhmister@ukr.net)

Article submitted 2021-07-30. Resubmitted 2021-09-14. Final acceptance 2021-09-17. Final version published as submitted by the authors. 